\title{
New future of cell biology and toxicology: thinking deeper
}

\author{
Jianying Gu • Xiangdong Wang
}

Received: 12 December 2015 / Accepted: 2 February 2016 / Published online: 13 February 2016

C) Springer Science+Business Media Dordrecht 2016

The combination of cell biology and toxicology (CBTo) demonstrates the potential effects of elements in cellular structures, organelles, functioning properties, metabolic circles, signaling pathways, or interactions with the microenvironment. These elements not only include chemicals and drugs, but one should also consider food, water, and even the air we breathe. Thus, a number of toxicology subspecialties are developed and will be furthermore defined with the development of biotechnologies and our further understanding of toxicogenomics and ecotoxicology or aquatic, chemical, clinical, environmental, forensic, medical, occupational, and regulatory toxicology. Thus, future issues of the journal will focus on clinical and translational research with an emphasis on molecular and cell biology, genetic and epigenetic heterogeneity, drug discovery and development, and molecular pharmacology and toxicology.

The merger of cell biology and toxicology enables a deeper insight of human disease-oriented and directed clinical research and science on gene- and protein-based regulation, cell type-specific function, and system

J. Gu

Department of Plastic Surgery, Zhongshan Hospital, Center of Biomedical Science Fudan University, Shanghai, China

X. Wang $(\triangle)$

Institute of Clinical Science, Zhongshan Hospital of Fudan

University, Shanghai, China

e-mail: xdwang@fuccb.com

X. Wang

Shanghai Institute of Clinical Bioinformatics, Shanghai, China biomedicine in drug discovery and development (Abraham, et al. 2012; Wang and Marincola. 2012). It is well accepted that "side effects" or "toxic effects" of elements from extracellular, intercellular, or intracellular resources contribute to the pathogeneses and exacerbations of diseases. During this process, we explore geneand protein-based regulations using genomics and proteomics, functional genomics and proteomics, computational biology, and gene expression and function analysis. The combination of cell biology and toxicology is a critical and important approach to figure out multifactorial genetics and epigenetics of diseases to develop the strategy of precision medicine and disease-specific biomarkers to monitor the deterioration of the disease and efficiency of therapies, developmental biology, and system biology to understand multi-dimensional principles of element actions or bioinformatics-based data mining approaches to paint the full picture of the disease. However, there is a considerable lack in our understanding of how an existing disease per se can reduce or increase the cell sensitivity and response to elements. For example, astrocytes as central nervous system glial cells in the disease change their biological behaviors and functions, resulting in the disorder of extracellular ion and neurotransmitter exchanges and dysfunction of the blood-brain barrier (Tangye et al. 2015). Pathological astrocytes will alter the diffusion speed of elements and reduce resistance of nervous cells, promoting and maintaining the "side effect."

The effect of combining the study of cell biology and toxicology to aid the identification of molecular targets during discovery and development of target-based drugs 
should be seriously considered. Drug target identification and validation is one of the most important steps during drug development and decides the processes of rational drug design, genetic/small molecule (highthroughput) screening, novel therapeutic approaches, combinatorial and parallel synthesis of drugs, pharmacokinetics and pharmacodynamics, pharmacogenomics, or toxicogenomics. Precision medicine has been suggested as a new approach to discovering and developing medicines and vaccines by integrating clinical and molecular information on molecular mechanism of diseases (Dolsten and Søgaard. 2012) to better select diseasespecific targets and identify patient populations and to improve clinical outcomes. The identification of disease-specific targets is of paramount importance in the development of precision medicine to deliver clinically significant treatments with favorable safety profiles. However, the behavior of targeting molecules may not be as simple as high or low expression levels, with or without mutations, or simply active or not. For example, CD5L/AIM was identified as a regulator expressed in non-pathogenic, but not in pathogenic Th17 cells by single-cell RNA-seq (Wang et al. 2015). CD5L could act as a functional switch to regulate Th17 cells from the normality to the pathogenicity which leads to the occurrence of autoimmunity, even though CD5L could not change Th17 differentiation. Thus, Dolsten and Søgaard (2012) recommended that the drug target should be the key driving factor to ensure the success of precision medicine. In addition, a number of important risk factors in the development of disease and mortality call for special attention from scientists when we measure the toxic effects of elements. In addition to elements per se, the combination of formulations, delivery forms and schedules, sleep-wake cycles, physical activities, related organ functions, sensitivity/resistance, lifestyle, or gut microbiome may add more on the variants between individuals, organs, cells, or molecules.

The influence of cell biology and toxicology on biological function- and disease-specific biomarkers not only for monitoring drug efficacy and efficiency but also for detecting drug toxicity and toxicology should be further considered. Disease-specific biomarkers have been suggested as one of five critical factors in the practice of precision medicine to monitor the quality of therapeutic strategies, to detect patient response to targeted treatments, and to predict the prognosis of disease (Chen et al. 2015; Wu et al. 2014). Dynamic changes of complex interaction networks and molecular subnetworks can represent and influence responses of cells or organs to real-time changed elements. We should pay even more attention on the identification and validation of biomarkers, network biomarkers, and dynamic network biomarkers to monitor drug resistance, toxicity or toxicology, by integrating bioinformatic-based screening with clinical informatics and phenotypic information (Zeng et al.; 2014; He et al. 2015). The rewiring of module networks is used to characterize functional reorganization of a complex biological function and to study the dynamical sensitivity and resistance of drugs. Consistent module genes can be directly used to reveal new genotypes relevant to drug sensitivity, different from gene expressions. We suggest the hierarchical structures of the temporal module network as spatio-temporal biomarkers to monitor the efficacy, efficiency, toxicity, and resistance of the therapy.

We should also consider the effect of genetic and functional alterations of membrane biology and domains or associated receptor- and non-receptormediated signaling and transport proteins of the cell membrane, mitochondria, endoplasmic reticulum, Golgi apparatus, and other organelles in health and disease. Also, it is important to investigate the influence of gene mutations, epigenetics, RNA profile changes (e.g., microRNA, circular RNA, long-noncoding RNA), DNA sequencing, or heterogeneity (Alam et al. 2016) in order to understand cancer development, maintenance and treatment resistance, immune and inflammatory cell function, neuroscience, stem cell development and differentiation, epithelial biology, and endothelial barrier function in toxicology of elements inducing carcinogenesis or treating cancer. For example, microRNAs control gene expression post-transcriptionally, are released upon cellular damage, and act as epigenetic regulators or potential non-invasive biomarkers of injury (Montgomery and Ruvkun. 2013). It is envisaged that combining cell biology and toxicology will make great contributions in the discovery and validation of miRNA biomarkers, provide an insight into injury mode of action, as well as, help to identify specific injury locations and affected cell types. Biological function- and disease-specific biomarkers should have a global standard for practice of toxicity and toxicology, for clinical validation, or for safety assessment. With the development of deep-sequencing approaches, altered lengths of miRNAs were suggested as critical factors regulating the processing to fine-tune miRNA target specificity (Yates et al. 2013). The altered miRNA seed 
sequence could guide strand selection to increase the number of targets a single pre-miRNA may eventually repress.

We should consider the effects of toxic elements produced from host cells during stress, injury, and trauma. Eosinophils play critical roles in the maintenance of innate immunity and the pathogenesis of various inflammatory and allergic disorders. During inflammatory responses, eosinophils can generate secretory granules which contain the eosinophil major basic protein, one of four cytotoxic proteins. Such protein toxicity can be activated by granule acidification and aggregated in extracellular microenvironment to mediate the damage to pathogens and regulate toxicity intra- and extracellularly (Soragni et al. 2015). This is important for innate immunity and immunopathology through its polymorphic self-association pathways. The overproduction of those toxic proteins may increase the sensitivity of cells; amplify toxic effects of elements in the immune system, central and autonomic nervous systems, gastrointestinal system, cardiovascular and circulatory system, endocrine system, pulmonary system, skeletal and muscular system, and urinary system; and help us to further understand their pathogeneses and therapies. In addition, we should consider the heterogeneity between individuals, organs/tissues, locations within the tumor, or cells within the location to respond to toxic elements.

Thus, it is the time to seriously consider the relationship between cell biology and toxicology. We have sufficient ways to investigate the alterations of spatial genome organization and their effects on transcription in cell toxicology, adopt the advanced chromatin interaction analysis by paired-end tag sequencing strategy, or understand higherorder chromosome folding and specific chromatin interactions in the topological mechanism of human variation and disease induced by toxic elements. We believe that singlecell genomics and biology will help us to understand the cell heterogeneity and response to elements, the molecular underpinnings at a genomic scale, or the molecular mechanisms for the heterogeneity and pathogenicity of toxics.

\section{References}

Abraham E, Marincola FM, et al. Clinical and translational medicine: integrative and practical science. Clin Transl Med. 2012;1:1.

Alam MM, Lal S, FitzGerald KE, Zhang L. A holistic view of cancer bioenergetics: mitochondrial function and respiration play fundamental roles in the development and progression of diverse tumors. Clin Transl Med. 2016;5:3.

Chen C, He M, et al. Five critical elements to ensure the precision medicine. Cancer Metastasis Rev. 2015;34(2):313-8.

Dolsten M, Søgaard M. Precision medicine: an approach to R\&D for delivering superior medicines to patients. Clin Transl Med. 2012;1:7.

He MY, Xia JL, et al. The development of precision medicine in clinical practice. Clin Transl Med. 2015;4:28.

Montgomery TA, Ruvkun G. MicroRNAs visit the ER. Cell. 2013;153(3):511-2.

Soragni A, Yousefi S, et al. Toxicity of eosinophil MBP is repressed by intracellular crystallization and promoted by extracellular aggregation. Mol Cell. 2015;57(6):1011-21.

Tangye SG, Brink R, et al. SnapShot: interactions between B cells and T cells. Cell. 2015;162(4):926-6.

Wang C, Yosef N, et al. Single-cell genomics unveils critical regulators of Th17 cell pathogenicity. Cell. 2015;163(6): 1400-12.

Wang XD, Marincola FM. A decade plus of translation: what do we understand? Clin Transl Med. 2012;1:3.

$\mathrm{Wu}$ XD, Chen LN, et al. Network biomarkers, interaction networks and dynamical network biomarkers in respiratory diseases. Clin Transl Med. 2014;3:16.

Yates LA, Norbury CJ, et al. The long and short of microRNA. Cell. 2013;153(3):516-9.

Zeng T, Wang DC, et al. Prediction of dynamical drug sensitivity and resistance by module network rewiring-analysis based on transcriptional profiling. Drug Resist Updat. 2014;17(3):64-76. 